\title{
Combined versus single antituberculosis drugs on the in vitro sensitivity patterns of non-tuberculous mycobacteria
}

\author{
J BANKS, P A JENKINS \\ From the Mycobacterium Reference Unit, Public Health Laboratory Service, Cardiff
}

ABSTRACT Drug sensitivity tests were performed for ethambutol, rifampicin, streptomycin, and? isoniazid both alone and in paired combinations, on 16 strains of Mycobacterium avium intra- $\vec{z}$ cellulare, seven strains of Mycobacterium xenopi, and eight strains of Mycobacterium malmoense. $Z$ Most strains were resistant to the individual drugs, but all strains of $M$ malmoense, $86 \%$ of $M \Phi$ xenopi, and $31 \%$ of $M$ avium intracellulare were completely suppressed by the lowest concentrations 0 of ethambutol and rifampicin when the two drugs were combined in vitro. Streptomycin combined with ethambutol or with rifampicin in the lowest combined concentrations suppressed $50 \%$ ando $62 \%$ respectively of strains of $M$ malmoense. All strains of $M$ xenopi were suppressed by the lowest combined concentrations of streptomycin with rifampicin. Combinations with isoniazid were less: effective. It is postulated that similar effects in vivo might account for the satisfactory clinicals response seen in patients with disease caused by these mycobacteria who have received treatmento with combinations of standard antituberculosis drugs despite in vitro resistance to the individua agents.

\section{Introduction}

The response to treatment with antituberculosis drugs is less predictable in patients with pulmonary infection caused by non-tuberculous mycobacteria than in those with disease caused by Mycobacterium tuberculosis. Serious coexistent pulmonary disease or compromised immunity undoubtedly contribute to the poor prognosis in many patients. In others, however, treatment with toxic second line drugs, given in regimens tailored to the results of in vitro sensitivity tests, has led to poor compliance by the patient and hence treatment failure. ${ }^{1}$ Decisions about treatment are complicated by the usual reports of in vitro resistance to first line drugs, which is characteristic of many of these organisms. Paradoxically, prolonged treatment with combinations of standard antituberculosis drugs is often successful despite in vitro resistance to the individual agents. ${ }^{23}$ The reason for this is not clear, although the discrepancy may reflect the limitations of the method used for sensitivity testing, which uses

Address for correspondence: Dr J Banks, Newport Chest Clinic, 129 Stow Hill, Newport, Gwent NP9 4GA. (Reprints will not be available.)

Accepted 5 June 1987 the concept of modal resistance. An alternative explanation is that drugs given in combination interact, and organisms resistant to the individual drugs become susceptible to their combined actions. $\overrightarrow{0}$ To investigate this hypothesis we have studied the effect of combining pairs of standard drugs in vitro on the sensitivity patterns of strains of Mycobacterium 3 . malmoense, Mycobacterium xenopi, and Mycobacterium avium intracellulare.

\section{Methods}

Sensitivity tests were performed on 16 strains of $M$ avium intracellulare, seven strains of $M$ xenopi, and $N$ eight strains of $M$ malmoense. Twenty of the strains N had originally been cultured from sputum specimens N and sent to the Mycobacterium Reference Unit in ${ }_{\sigma}^{\omega}$ Cardiff. Another six strains of $\boldsymbol{M}$ avium intracellulare were cultured from resected lymph nodes, another two from lung sections taken at necropsy, and one other from pleural fluid.

Routine techniques for the examination of strains, $\frac{0}{0}$ as described by Marks, ${ }^{4}$ were used throughout the $\widetilde{\Phi}$ study. Briefly, strains were subcultured on slopes of $\frac{?}{\mathbb{Q}}$ glycerol egg medium, and after three weeks incu- $\varrho$ bation suspensions were made by transferring a $2 \mathrm{~mm}<$ 
loopful of growth into screw capped bottles contain-

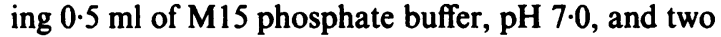
nails. After being stirred on a magnetic stirrer the resultant suspension was diluted 1:5 in sterile water and then inoculated in $3 \mu \mathrm{l}$ aliquots on to control and drug slopes. These were incubated in the dark at $37^{\circ} \mathrm{C}$ for three weeks.

Sensitivity tests against single drugs - ethambutol, rifampicin, streptomycin, and isoniazid-were performed in the usual way by the technique of modal resistance. $^{45}$ Tubes containing doubling concentrations of each drug were inoculated with the test strains and incubated in the dark at $37^{\circ} \mathrm{C}$ for three weeks. The drug concentrations used (table 1) were derived by first determining the normal range of tolerance of several standard strains of $M$ tuberculosis to each drug and accepting the most frequent end point of the titration as the "mode." Doubling concentrations above the mode were then incorporated into tubes numbered 2, 4 and 8 for testing strains of $M$ malmoense and $M$ xenopi. The range was extended to include tubes 16, 32, and 64 for testing strains of $M$ avium intracellulare, which were highly resistant to the individual drugs.

Sensitivity testing for drug combinations was performed according to a chequerboard design, so that for each pair of drugs all possible permutations of the concentrations used for testing against single agents were tested for strains of $M$ malmoense and $M$ xenopi. Not all permutations were tested for strains of $M$ avium intracellulare because of the large number of combinations that this would have required.

In a second experiment sensitivity tests for rifampicin and ethambutol, both alone and in combination, were repeated for five of the strains of $M$ malmoense and five of the strains of $M$ xenopi. Tubes with drug concentrations corresponding to resistance ratios of $\frac{1}{2}$ and 1 were included on this occasion.

The results were read after three weeks' incubation. Each tube was examined with a hand lens for evidence

Table 1 Drug concentrations used for in vitro sensitivity tests

\begin{tabular}{lllll}
\hline \multirow{2}{*}{$\begin{array}{l}\text { Resistance } \\
\text { ratio }\end{array}$} & \multicolumn{4}{l}{ Drug concentrations in culture medium $(\mu \mathrm{g} / \mathrm{ml})^{*}$} \\
\cline { 2 - 5 } & Rifampicin & Ethambutol & Streptomycin & Isoniazid \\
\hline$\frac{1}{2}$ & 4 & 0.4 & 2.5 & 0.01 \\
1 & 8 & 0.8 & 5 & 0.02 \\
2 & 16 & 1.6 & 10 & 0.05 \\
4 & 32 & 3.2 & 20 & 0.11 \\
8 & 64 & 6.4 & 40 & 0.22 \\
16 & 128 & 12.8 & 80 & 0.44 \\
32 & 256 & 25.6 & 160 & 0.89 \\
64 & 512 & 51.2 & 320 & 1.7 \\
\hline
\end{tabular}

* Concentrations corresponding to resistance ratios of 2-8 for standard strains of $M$ ycobacterium tuberculosis were used for testing strains of $M$ malmoense and $M$ xenopi and resistance ratios 2-64 for strains of $M$ avium intracellulare. of bacterial growth and compared with the control tubes. Absence of visible bacterial colonies was accepted as the end point, although small discrete specks were regarded as representing a negative result.

\section{Results}

The results of the sensitivity tests for both single drugs and drug combinations are shown in tables 2-4. The percentage of strains that grew in tubes containing single drugs are shown adjacent to the tube numbers and the percentage of strains that grew in tubes containing drug combinations are entered on the chequerboard.

The overall results show that drugs in combination were more effective than the individual agents in suppressing bacterial growth.

\section{$M$ malmoense (table 2)}

Rifampicin combined with ethambutol completely suppressed all strains of $M$ malmoense even at the lowest combined concentrations (tube $2 \times 2$ ), although $88 \%$ of strains were resistant to the same concentrations of the individual drugs. Combinations of ethambutol or rifampicin with streptomycin were more effective than the individual drugs alone. All strains of $M$ malmoense were highly resistant to isoniazid, and susceptibility to either rifampicin, ethambutol, or streptomycin was unchanged or differed by only one tube when the drugs were combined with isoniazid (results not shown).

Table 2 Mycobacterium malmoense: percentage growth on slopes*

\begin{tabular}{|c|c|c|c|c|c|c|c|c|c|}
\hline & \multirow{2}{*}{$\begin{array}{l}\text { Tube } \\
\text { No }\end{array}$} & \multicolumn{4}{|c|}{ Rifampicin } & \multicolumn{4}{|c|}{ Streptomycin } \\
\hline & & 0 & 2 & 4 & 8 & 0 & 2 & 4 & 8 \\
\hline \multirow{4}{*}{ 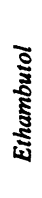 } & 0 & 100 & 88 & 75 & 38 & 100 & 88 & 88 & 38 \\
\hline & 2 & 88 & 0 & 0 & 0 & & 50 & 25 & 0 \\
\hline & 4 & 50 & 0 & 0 & 0 & & $\mathbf{0}$ & $\mathbf{0}$ & 0 \\
\hline & 8 & 0 & 0 & 0 & 0 & & 0 & 0 & 0 \\
\hline \multirow{4}{*}{ 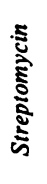 } & 0 & 100 & & & & & & & \\
\hline & 2 & 88 & 38 & 25 & 0 & & & & \\
\hline & 4 & 88 & 25 & 12 & 0 & & & & \\
\hline & 8 & 38 & 0 & 0 & 0 & & & & \\
\hline
\end{tabular}

*The percentage of strains that grew on slopes inoculated with the single drugs are shown within the margins, and the percentage that grew in tubes containing drug combinations are entered on the chequerboard. Tube numbers correspond to the resistance ratios shown in table 1, from which the respective drug concentrations used can be obtained. 
$M$ xenopi (table 3)

All strains of $M$ xenopi were resistant to the concentrations of ethambutol in tube 4 and $72 \%$ were resistant to rifampicin in tube 2 . All strains, however, were suppressed by the same concentrations of the two drugs combined. Streptomycin combined with rifampicin was effective against all strains of $M$ xenopi at the lowest combined concentration. Seventy two per cent of strains were resistant to isoniazid in tube 8 but all strains were suppressed by the same concentration of isoniazid combined with the concentration of rifampicin in tube 2 . Combinations of isoniazid with either ethambutol or streptomycin failed to alter the sensitivity results for the individual drugs (results not shown).

\section{$M$ avium intracellulare (table 4)}

Most strains of $M$ avium intracellulare were highly resistant to the individual drugs, although one strain (6\%) was suppressed by streptomycin in tube 4 . All strains were resistant to isoniazid in tube 64 (results not shown). Drugs in combination were more inhibitory than the single agents alone but failed to achieve the degree of growth inhibition seen with strains of $M$ malmoense and $M$ xenopi. Rifampicin combined with ethambutol produced the best results and in the lowest combined concentrations (tube

Table 3 Mycobacterium xenopi: percentage growth on slopes*

\begin{tabular}{|c|c|c|c|c|c|c|c|c|c|}
\hline & \multirow{2}{*}{$\begin{array}{l}\text { Tube } \\
\text { No }\end{array}$} & \multicolumn{4}{|c|}{ Rifampicin } & \multicolumn{4}{|c|}{ Streptomycin } \\
\hline & & 0 & 2 & 4 & 8 & 0 & 2 & 4 & 8 \\
\hline \multirow{4}{*}{ 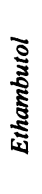 } & 0 & 100 & 72 & 43 & 0 & 100 & 29 & 14 & 0 \\
\hline & 2 & 100 & 14 & 14 & 0 & & 14 & 0 & 0 \\
\hline & 4 & 100 & 0 & 0 & 0 & & 14 & 0 & $\mathbf{0}$ \\
\hline & 8 & 29 & 0 & 0 & 0 & & 0 & 0 & $\mathbf{0}$ \\
\hline \multirow{4}{*}{ 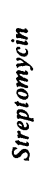 } & 0 & 100 & & & & & & & \\
\hline & 2 & 29 & 0 & 0 & 0 & & & & \\
\hline & 4 & 14 & $\mathbf{0}$ & 0 & 0 & & & & \\
\hline & 8 & $\mathbf{0}$ & $\mathbf{0}$ & 0 & 0 & & & & \\
\hline \multirow{4}{*}{ 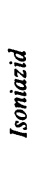 } & 0 & 100 & & & & & & & \\
\hline & 2 & 100 & 57 & 14 & 0 & & & & \\
\hline & 4 & 100 & 29 & 14 & 0 & & & & \\
\hline & 8 & 72 & 0 & 0 & 0 & & & & \\
\hline
\end{tabular}

* The percentage of strains that grew on slopes inoculated with the single drugs are shown within the margins, and the percentage that grew in tubes containing drug combinations are entered on the chequerboard. See table 1 to derive the drug concentrations used in the tubes, which are numbered according to resistance ratios.
$2 \times 2$ ) suppressed almost a third of strains that were resistant to the same concentrations of the individual $\frac{}{0}$ drugs. Sensitivity to rifampicin, streptomycin, and $\frac{\bar{\sigma}}{\mathrm{m}}$ ethambutol was virtually unchanged by their combi- $\vec{\Phi}$ nation with isoniazid (results not shown).

Rifampicin and ethambutol: second experiment (figs $1 \vec{\circ}$ and 2)

There were no significant changes in the sensitivity $\vec{\omega}$ results for rifampicin or ethambutol when the tests $\underset{\vec{F}}{\overrightarrow{2}}$ were repeated with strains of $M$ malmoense and $M$

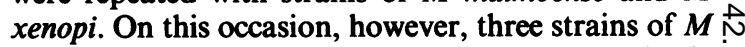
malmoense and four of $M$ xenopi were completely $\vec{A}$ suppressed by the two drugs when combined in concentrations that corresponded with modal resistances $\infty$ of $\frac{1}{2}$ for each drug. The results are shown as iso- 음 bolograms (figs 1 and 2). A line or isobol is drawn for each strain that joins the minimum effective concen- $z$ tration of ethambutol when used alone (on the ordinate) with the minimum effective concentration of 3 rifampicin when used alone (on the abscissa) through $\mathbb{\Phi}$ the point representing their minimum effective con- $\vec{P}$ centrations when combined. The inset shows three $\infty$ theoretical isobols. Drug combinations that are syn-.ergistic produce concave isobols (ESR), antagonistic combinations produce convex isobols (EAR), while additive combinations produce straight lines (EXR). ${ }^{6}$ Each strain of $M$ malmoense and $M$ xenopi produced a concave isobol, consistent with a synergistic effect $\stackrel{\Phi}{\complement}$ between rifampicin and ethambutol.

\section{Discussion}

The patterns of resistance to the major antituberculosis drugs in this study are characteristic of $\frac{O}{7}$ the three mycobacterial species studied, which are $\times$ often fully sensitive in vitro only to second line $\frac{\sigma}{3}$ agents - for example, cycloserine and ethionamide. Treatment regimens tailored to these results have not, however, been generally successful, probably because of drug toxicity and poor compliance by patients. ${ }^{1-3}$ 윽 In contrast, other patients have been successfully $\frac{}{O}$ treated with combinations of standard drugs despite in vitro resistance..$^{1-3}$ The reason for this paradox is not clear, although the design of the sensitivity test or, alternatively, drug interactions in vivo may account

Drug susceptibility tests for both $M$ tuberculosis and non-tuberculous mycobacteria are performed byo the method of modal resistance. ${ }^{45}$ This compares the sensitivity of the test strain with the modal sensitivity? of several standard strains of $M$ tuberculosis and the $\underset{T}{T}$ result is expressed as a ratio of the test strain's sensitivity to the mode. Conventionally, strains that are $\bigcirc$ suppressed in tubes 2 or 4 are reported as sensitive $\mathbb{Q}$ and those that grow in tube 4 but not in tube 8 are 
Table 4 Mycobacterium avium intracellulare: percentage growth on slopes*

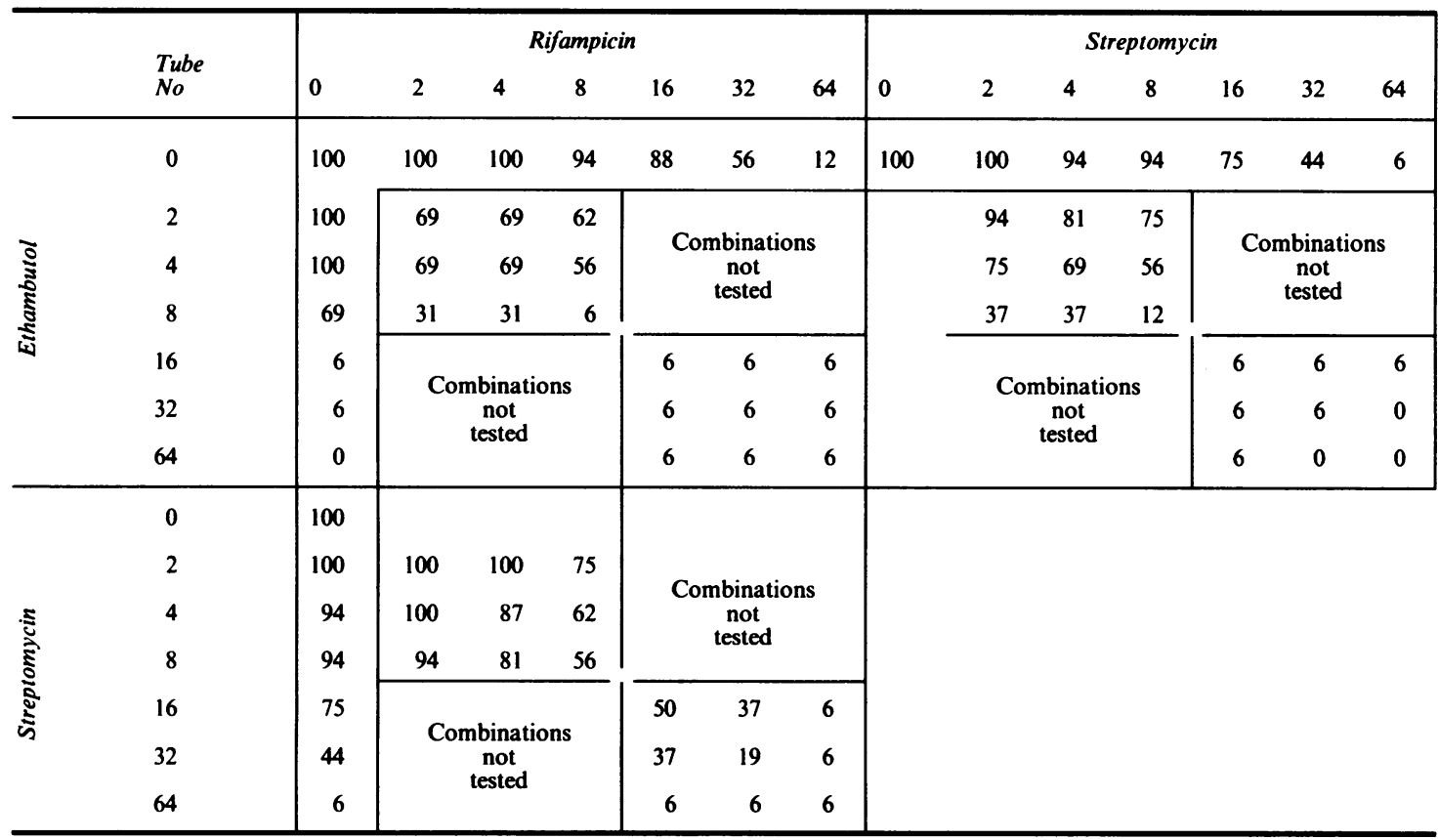

*The percentage of strains that grew on slopes inoculated with the single drugs are shown within the margins, and the percentage that grew in tubes containing drug combinations are entered on the chequerboard. See table 1 to derive the drug concentrations used in the tubes, which are numbered according to resistance ratios.

$M$ malmoense (2nd experiment)

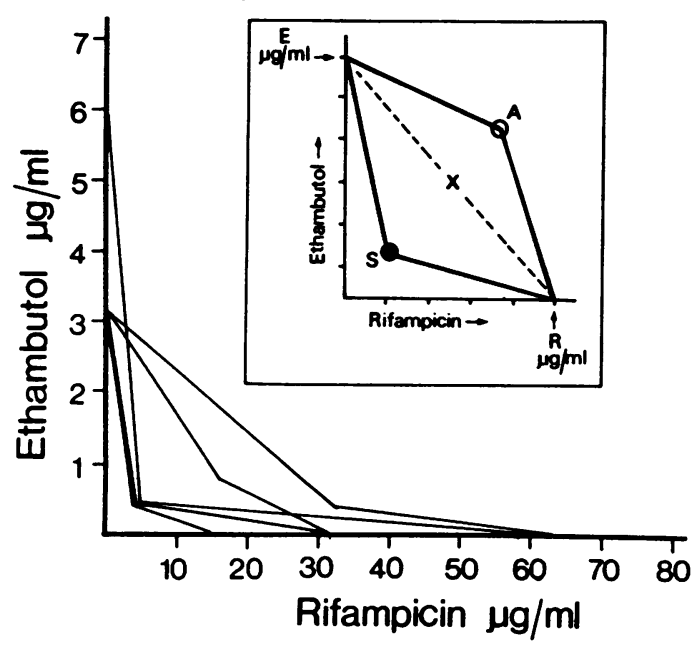

Fig 1 Isobolograms for strains of Mycobacterium malmoense tested against ethambutol combined with rifampicin (see text for explanation of inset). reported as resistant with a ratio of 4 . Strains that grow in tube 8 - that is, having resistance ratio 8 -are reported as highly resistant. The clinical significance of any differences from the mode has to be established for each drug by correlation with the clinical response to treatment. For strains of $M$ tuberculosis any decline from modal sensitivity is detrimentalthat is, even low degrees of resistance are clinically important. ${ }^{5}$ The same criteria may not, however, apply to non-tuberculous mycobacteria, for which comparable degrees of resistance may have no clinical importance. Nevertheless, the results are reported in the same way. The modal resistance method does not relate sensitivity to drug concentrations attained in the blood or tissues of patients, and organisms with a resistance ratio of 4 , for example, for ethambutol are still suppressed in vitro by drug concentrations that can be achieved in vivo with conventional doses. Tailoring treatment according to the modal resistance method makes the assumption that resistance criteria applicable to strains of $M$ tuberculosis are equally applicable to non-tuberculous mycobacteria. This assumption may be incorrect.

Alternatively, the effectiveness of standard drug regimens in treatment may be due to drug interaction 


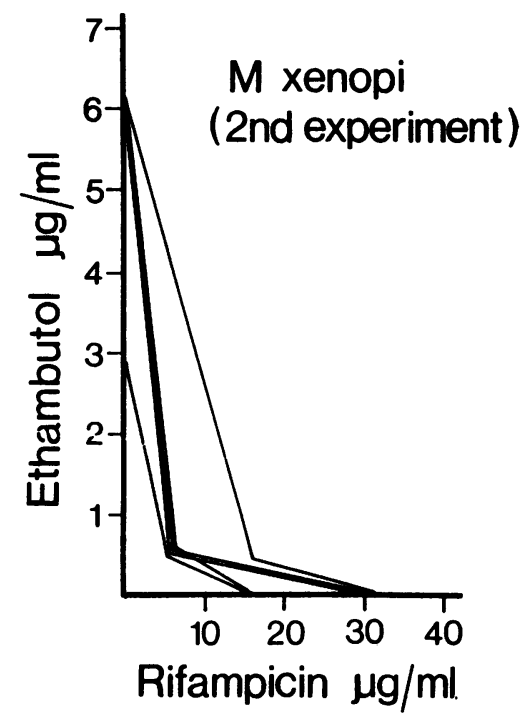

Fig 2 Isobolograms for strains of Mycobacterium xenopi tested against ethambutol with rifampicin.

in vivo. In this study the enhanced effect of drug combinations in vitro on mycobacteria resistant to individual drugs supports this idea. Experimental conditions may not imitate in vivo conditions and factors such as protein binding may modify the actions of drugs used in treatment. Nevertheless, the results correlate well with the clinical response to treatment. The particular efficacy of the rifampicin and ethambutol combination was not due to any reduction in the degree of protein binding of rifampicin in vitro caused by the addition of ethambutol. We have found that the free non-protein bound fraction of rifampicin is the same whether it is used alone or combined in vitro with ethambutol (unpublished observations). The effect appears to be due to a genuine interaction between the two agents and was reproducible, even at low drug concentrations. Rifampicin combined with ethambutol has been effective in patients with $M$ malmoense infection, and withdrawal of ethambutol from such regimens has $\frac{\bar{n}}{\overline{2}}$ been followed by clinical deterioration and bacte- $-\overline{\frac{p}{}}$

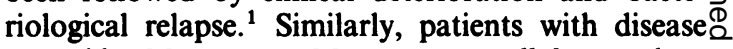
caused by $M$ xenopi or $M$ avium intracellulare are best treated with regimens of standard antituberculosis drugs. $^{2} 3$

Because of their clinical and radiographic abnor $-\overrightarrow{\vec{\omega}}$

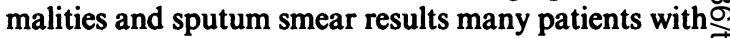
non-tuberculous mycobacterial disease are initially $\vec{x}$ misdiagnosed as having classical $\boldsymbol{M}$ tuberculosis and started on treatment with standard antituberculosis ${ }_{-}^{-}$ drugs. In most of them treatment will be successful. despite the subsequent reports of in vitro resistance to $\mathbb{\infty}_{\infty}$ one or more of the individual drugs prescribed. Alter- $\circ$ ations to such successful regimens made on the basis ${ }_{-}^{-}$ of the sensitivity results should be avoided. Sensitivity $\vec{Z}_{Z}$ testing of drug combinations might yield results thato agree more closely with clinical response but a pros- $\frac{\Phi}{7}$ pective study is needed to confirm this.

We wish to thank the staff of the Public Health $\overrightarrow{0}$ Laboratory Service, Mycobacterium Reference Unit,, Cardiff, for their technical assistance and Mrs $S_{\square}$ Workman for typing the manuscript.

\section{References}

1 Banks J, Jenkins PA, Smith AP. Pulmonary infection $\stackrel{\odot}{\triangle}$ with Mycobacterium malmoense - a review of treat- $\vec{\partial}$ ment and response. Tubercle 1985;66:197-203.

2 Banks J, Hunter AM, Campbell IA, Jenkins PA, Smith AP. Pulmonary infection with Mycobacterium xenopi: review of treatment and response. Thorax 1984;39:

3 Hunter AM, Campbell IA, Jenkins PA, Smith AP. 을 Treatment of pulmonary infection caused by mycobacteria of the Mycobacterium avium- 0 intracellulare complex. Thorax 1981;36:326-9.

4 Marks J. A system for the examination of tubercle bacillio and other mycobacteria. Tubercle 1976;57:205-7.

5 Marks J. The design of sensitivity tests on tubercle bacilli. Tubercle 1961;42:314-6.

6 Berenbaum MC. A method for testing for synergy with any number of agents. J Infect Dis 1978;137:122-30. 